\title{
Funcionalidade de membros inferiores em vítimas de acidentes com motocicleta
}

\author{
Functionality of lower limbs in victims of accidents \\ with motorcycle
}

\author{
Camila Câmara Reis ${ }^{1}$ \\ Fernanda Fonsêca Fernandes² \\ Saionara Maria Aires da Câmara ${ }^{3}$ \\ Sanny de Aquino Ferreira 4 \\ Álvaro Campos Cavalcanti Maciel ${ }^{5}$
}

\section{RESUMO}

Objetivo: Analisar a funcionalidade dos membros inferiores em vítimas de acidentes de trânsito com motocicletas no município do Natal/RN. Metodologia: Em estudo transversal, 52 acidentados de moto com fratura em membros inferiores foram avaliados quanto ao acidente e funcionalidade de membros inferiores pela LEFS. Resultados: A maioria dos acidentados era do sexo masculino $(75,0 \%)$, com média de idade de 32,5 $( \pm 10,2)$ anos. A média do escore da LEFS foi de 31,3 $( \pm 10,2)$ pontos. Piores resultados foram encontrados para mulheres, pessoas que não faziam revisões na moto, que relataram ter ficado com sequelas pós-acidente e que sofreram o acidente mais recentemente. Permaneceram com significância após análise ajustada as variáveis sexo, presença de sequela e tempo do acidente $\left(R^{2}=0,40\right)$. Conclusões: Devido ao seu impacto na saúde das vítimas, se faz necessário maior atenção pós-acidente, propiciando uma reabilitação precoce, além do desenvolvimento de estratégias de conscientização e prevenção de acidentes com motocicletas.

\section{PALAVRAS-CHAVE:}

Lesões, Reabilitação, Extremidade Inferior.

\footnotetext{
1,2,4 Universidade Federal do Rio Grande do Norte.

${ }^{3}$ Doutora em Fisioterapia, Professora do Curso de Fisioterapia da Faculdade de Ciências da Saúde do Trairi, da Universidade Federal do Rio Grande do Norte.

${ }^{5}$ Doutor em Ciências da Saúde, Professor do Curso de Fisioterapia e do Programa de Pós-Graduação em Fisioterapia da Universidade Federal do Rio Grande do Norte.
} 


\section{ABSTRACT}

Objective: To analyze the lower limbs functionality in victims of traffic accident with motorcycle in the city of Natal/RN. Materials and Methods: It was performed a cross-sectional study with 52 patients, victims of motorcycle accident, which presented fracture of the lower limbs. The assessment was performed through a questionnaire containing aspects related to the accident and evaluation of the functionality of the lower limbs (LEFS). Results: Most victims were male

(75.0\%), with a mean of $32.5( \pm 10.2)$ years old. The LEFS score average was $31.2( \pm 10.2)$ points. There was a statistic significant difference between independent variables: gender $(p=0.004)$, perform reviews on the motorcycle $(p=0.001)$, presence of physical sequel after accident $(p=0.007)$ with LEFS scores and positive correlation between LEFS and the time of accident $(r=0.39 ; p=0.02)$. Worst results were found for women, people that did not perform reviews on the motorcycle, people that report to be with sequels and for those that suffered accident more recently. The variables that remained with statistical significance were gender, presence of sequel e time of the accident $\left(R^{2}=0,40\right)$. Conclusions: Due to the impact in the victims' health, more attention is needed the period after accident, providing an early rehabilitation and development of strategies to increase awareness and prevention of motorcycle accidents.

\section{KEYWORDS:}

Injuries, Rehabilitation, Lower Extremity. 


\section{INTRODUÇÃO}

O Brasil, nas últimas décadas, vem aos poucos se tornando um dos países com os mais altos índices de acidentes de trânsito em virtude de vários motivos, como o aumento no número de veículos em circulação nas ruas, a imprudência dos condutores, a ausência de fiscalização e punição e o tráfego desorganizado ${ }^{1,2}$. Sendo assim, o país é considerado como um dos piores e mais perigosos trânsitos do mundo ${ }^{3}$.

Entre esses acidentes, merece destaque aqueles que envolvem motocicletas, visto que esse meio de transporte está cada vez mais sendo utilizado e aprovado pela população, por ser ágil e financeiramente mais viável ${ }^{1,4,5}$. Outro fator que também colabora para o aumento dos acidentes motociclísticos é a maior vulnerabilidade do condutor e também do passageiro à lesões graves, em virtude destes se encontrarem bem mais expostos do que motoristas de automóveis ${ }^{2,6-8}$. Não obstante, as atitudes inadequadas dos condutores de motocicletas ${ }^{2,4,9}$ e a difícil visualização dos motociclistas no trânsito agravam essa situação ${ }^{4,9}$.

Segundo Koizumi ${ }^{10}$, a proporção de feridos nos acidentes com motocicletas é muito maior do que aqueles que envolvem automóveis. Enquanto 71\% dos acidentes com motocicletas envolvem feridos que necessitam de cuidados hospitalares, esse percentual cai para $7 \%$ quando envolvem outros meios de transporte, segundo dados do Instituto de Pesquisa Econômica Aplicada"1.

Entre as vítimas de acidentes de moto, a maioria é jovem e do sexo masculino ${ }^{6-8,12-14}$, fato este que conduz a altas taxas de morbi-mortalidade nessa camada da população, os quais se encontram no auge de suas capacidades laborais, acarretando consideráveis danos à economia brasileira ${ }^{12}$.

Ao considerar os tipos de lesões e áreas corporais mais frequentemente atingidas, as fraturas em extremidades estão entre os principais ${ }^{5,8,10,12,3}$. Lesões isoladas em membros superiores ou inferiores raramente evoluem para óbito, porém, com frequência, estão relacionadas à realização de cirurgias corretivas, reparadoras e até mesmo amputações que alteram a qualidade de vida tanto dos pacientes quanto de seus familiares ${ }^{15,16}$. Além disso, podem desencadear imobilizações prolongadas, acarretando longos períodos de recuperação à vítima ${ }^{2}$.

Tendo em vista que os membros inferiores são de fundamental importância para a biomecânica corporal, principalmente durante a realização da marcha, uma vez comprometidos, poderão levar à perda de capacidade funcional importante, acarretando dificuldade na realização das atividades de vida diária. Nos casos mais graves, pode ser necessário o uso de dispositivos auxiliares para se deslocar, tais como muletas e cadeiras de rodas. Além disso, há o comprometimento do equilíbrio que pode culminar em quedas frequentes ou medo de cair, limitando assim a deambulação ${ }^{17}$.

Diante do exposto, este estudo objetiva caracterizar o perfil sócio-demográfico de vítimas por acidentes de trânsito com motocicletas no município do Natal/RN, analisar as alterações funcionais provenientes de fraturas dos membros inferiores e identificar as variáveis associadas a essas alterações.

\section{METODOLOGIA}

\section{Caracterização do estudo}

Trata-se de um estudo epidemiológico de caráter transversal, com pacientes acidentados de motocicleta, realizado no Hospital Monsenhor Walfredo Gurgel (HMWG), em Natal/RN. O estudo foi aprovado no Comitê Ética do Hospital Universitário Onofre Lopes (HUOL), sob parecer número 625/2011, de acordo com a Resolução 196/96, do Conselho Nacional de Saúde.

\section{População e Amostra}

A população foi composta de indivíduos de ambos os sexos, com idade acima de 18 anos, vítimas de acidente de trânsito com motocicletas e que sofreram fratura em membros inferiores decorrente do acidente, atendidos no HMWG.

A amostra foi constituída pelos indivíduos que sofreram acidente de trânsito com motocicletas e que apresentassem fratura nos membros inferiores. A avaliação foi feita no período de um mês até seis meses após o acidente. Esse período foi estabelecido para que se pudesse verificar a influência do tempo na recuperação desses indivíduos. 
Aqueles que tiveram lesão do tipo traumatismo crânio-encefálico (TCE) em virtude do acidente foram excluídos da amostra devido aos possíveis comprometimentos neurológicos e cognitivos, o que poderia dificultar a aplicação do protocolo de avaliação.

Os indivíduos foram informados sobre os objetivos do estudo, caráter metodológico dos procedimentos a que foram submetidos e assinaram um termo de consentimento livre e esclarecido no momento da aplicação do questionário e do protocolo de avaliação clínica e funcional dos membros inferiores, de acordo com a resolução CNS 196/96.

\section{Instrumentos}

Foi utilizado um questionário estruturado contendo dados de identificação como: nome, endereço, telefone, data de nascimento, sexo, estado civil, escolaridade, religião, naturalidade, profissão, se o condutor tinha habilitação e há quanto tempo, quantas cilindradas a moto possuía, e também questões relacionadas ao acidente como: data do evento traumático, áreas corporais em que houve fratura, existência de sequelas, tempo de hospitalização, realização de cirurgias, tratamento fisioterapêutico, consumo prévio de álcool, uso de capacete, tipo de acidente e se já sofreu acidentes anteriores.

Também foi aplicada a escala: The Lower Extremity Funcional Scale (LEFS) ${ }^{18}$ para avaliação dos aspectos funcionais dos membros inferiores. O questionário é composto por vinte questões sobre capacidade em realizar atividades diárias. Os participantes deveriam responder qual o nível de dificuldade percebiam para executá-las e assim obtinham a pontuação correspondente a: $0=$ Extrema dificuldade ou incapacidade, $1=$ Muita dificuldade, $2=$ Dificuldade moderada, $3=$ Pouca dificuldade e $4=$ Nenhuma dificuldade.

As respostas foram somadas para gerar uma pontuação que variou de 0 a 80 pontos, com zero indicando altos níveis de limitação da atividade e 80 indicando baixos níveis de limitação da atividade.

\section{Análise estatística}

A construção do banco de dados e a análise estatística foram feitas no programa estatístico SPSS, versão 17.0 for Windows.

Na análise descritiva as variáveis quantitativas foram apresentadas por média e desvio-padrão $(d p)$, enquanto os dados categóricos apresentados na for- ma de tabela, por frequências absolutas e relativas. Para a comparação das médias dos escores da LEFS entre as variáveis categóricas foi utilizado o teste $t$ de Student para amostras independentes. Além disso, utilizou-se o teste de Correlação de Pearson.

Por fim, para analisar as relações entre o escore da LEFS com as variáveis independentes foram construídos modelos de regressão linear múltiplo, com o objetivo de identificar o grau de predição e com isso, determinar qual aquela que mais influenciou linearmente nos resultados. Em toda a análise foi considerado um $p$ valor padrão de 0,05 e intervalo de confiança de $95 \%$.

\section{RESULTADOS}

A amostra foi composta por 52 indivíduos (média de idade de 32,5 ), que sofreram fratura em membros inferiores. A maioria era do gênero masculino, não eram casados nem possuíam união estável, possuíam carteira de habilitação, sendo habilitados há mais de 7 anos em média (tabela 1).

Tabela 1- Caracterização da amostra em relação ao acidentado, Natal, RN, Brasil, 2011.

\begin{tabular}{|c|c|c|}
\hline VARIÁVEIS & n (\%) & Média $( \pm d p)$ \\
\hline Idade (anos) & & $32,5( \pm 10,2)$ \\
\hline \multicolumn{3}{|l|}{ Sexo } \\
\hline Masculino & \multicolumn{2}{|l|}{$39(75,0)$} \\
\hline Feminino & $13(25,0)$ & \\
\hline \multicolumn{3}{|l|}{ Estado Civil } \\
\hline $\begin{array}{l}\text { Casado/União Estável } \\
\text { Solteiro/Viúvo/ } \\
\text { Divorciado }\end{array}$ & $\begin{array}{l}21(38,5) \\
31(59,5)\end{array}$ & \\
\hline \multicolumn{3}{|l|}{ Habilitação } \\
\hline $\begin{array}{l}\text { Sim } \\
\text { Não }\end{array}$ & $\begin{array}{l}28(53,8) \\
24(46,2)\end{array}$ & \\
\hline \multicolumn{2}{|l|}{$\begin{array}{l}\text { Tempo de habilitação } \\
\text { (anos) }\end{array}$} & $7,5( \pm 5,4)$ \\
\hline
\end{tabular}

A tabela 2 mostra os dados em relação à moto e ao acidente. A maior parte dos indivíduos avaliados possuíam motocicleta com menos de 125 cilindradas, pilotavam moto há menos de quatro anos e estavam com as revisões da moto em dia na época do acidente. Para a maior parte dos avaliados, 0 acidente ocorreu nos turnos da tarde e da noite. A média da escala LEFS foi de $31,3( \pm 10,2)$. 
Tabela 2- Caracterização da amostra em relação ao acidente com motocicleta, Natal, RN, Brasil, 2011.

\begin{tabular}{|c|c|c|}
\hline VARIÁVEIS & $n *(\%)$ & Média $( \pm d p)$ \\
\hline \multicolumn{3}{|c|}{ Cilindradas da moto } \\
\hline $\begin{array}{l}\text { Menos de } 125 \mathrm{cc} \\
\text { Mais de } 125 \mathrm{cc}\end{array}$ & $\begin{array}{l}28(68,3) \\
14(31,7)\end{array}$ & \\
\hline \multicolumn{3}{|c|}{ Tempo de uso da moto } \\
\hline $\begin{array}{l}\text { Mais de } 4 \text { anos } \\
\text { Menos de } 4 \text { anos }\end{array}$ & $\begin{array}{l}19(35,0) \\
33(65,0)\end{array}$ & \\
\hline \multicolumn{3}{|l|}{ Revisões em dia } \\
\hline $\begin{array}{l}\text { Sim } \\
\text { Não }\end{array}$ & $\begin{array}{l}32(87,5) \\
8(12,5)\end{array}$ & \\
\hline \multicolumn{3}{|l|}{ Hora do acidente } \\
\hline $\begin{array}{l}\text { Manhã } \\
\text { Tarde } \\
\text { Noite }\end{array}$ & \begin{tabular}{|l|}
$16(30,8)$ \\
$18(34,6)$ \\
$18(34,6)$
\end{tabular} & \\
\hline \multicolumn{3}{|l|}{$\begin{array}{l}\text { Outros veículos } \\
\text { envolvidos }\end{array}$} \\
\hline $\begin{array}{l}\text { Sim } \\
\text { Não }\end{array}$ & $\begin{array}{l}30(57,7) \\
22(42,3)\end{array}$ & \\
\hline \multicolumn{3}{|l|}{ Fez cirurgia } \\
\hline $\begin{array}{l}\text { Sim } \\
\text { Não }\end{array}$ & $\begin{array}{l}39(76,5) \\
13(23,5)\end{array}$ & \\
\hline $\begin{array}{l}\text { Tempo para } \\
\text { cirurgia (dias) }\end{array}$ & & $10,8( \pm 9,3)$ \\
\hline $\begin{array}{l}\text { Tempo de } \\
\text { internação (dias) } \\
\text { Fez Fisioterapia }\end{array}$ & & $4,9( \pm 3,1)$ \\
\hline Sim & $18(35,3)$ & \\
\hline Não & $34(64,7)$ & \\
\hline \multicolumn{2}{|c|}{$\begin{array}{l}\text { Inicio da fisioterapia } \\
\text { após cirurgia (dias) }\end{array}$} & $61,2( \pm 18,1)$ \\
\hline \multicolumn{3}{|l|}{ Sequela Física } \\
\hline Sim & $44(86,0)$ & \\
\hline Não & $8(14,0)$ & \\
\hline \multicolumn{3}{|l|}{ Acidente anterior } \\
\hline Sim & $20(38,5)$ & \\
\hline Não & $32(61,5)$ & \\
\hline \multicolumn{3}{|l|}{ Com passageiro } \\
\hline Sim & $20(38,5)$ & \\
\hline Não & $32(61,5)$ & \\
\hline \multicolumn{3}{|c|}{ Uso de bebida alcoólica } \\
\hline Sim & $4(7,7)$ & \\
\hline Não & $48(92,3)$ & \\
\hline
\end{tabular}

* Apenas dos casos válidos
$\mathrm{Na}$ análise das variáveis independentes com os escores da LEFS, que determinam níveis de funcionalidade, pode-se observar que houve diferença estatisticamente significativa em relação ao sexo $(p=0,004)$, realização de revisões $(p=0,001)$ e presença de sequela física pós-acidente $(p=0,007)$, conforme a tabela 3 (na página seguinte). Além disso, encontrou-se correlação positiva apenas entre a LEFS e o tempo do acidente $(r=0,39 ; p=0,02)$.

$\mathrm{Na}$ análise de regressão linear múltipla, o escore total da LEFS permaneceu significativamente associado $\left(R^{2}=0,40 ;-=21,5\right)$ com o sexo, presença de sequela e o tempo do acidente, conforme a tabela 4 (na página seguinte).

\section{DISCUSSÃO}

Os acidentes de trânsito destacam-se pela sua magnitude, tanto em termos de mortalidade quanto de morbidade, sendo responsáveis pelo grande número de anos potenciais de vida perdidos, por limitações e incapacidades ${ }^{19-22}$.

Ao analisar o perfil da amostra, verificou-se que a maior parte das vítimas de acidentes com motocicletas na cidade do Natal/RN foram jovens do sexo masculino, o que corrobora com outros estudos realizados ${ }^{8,13,14}$. Pesquisas afirmam que a faixa etária de jovens está mais envolvida em acidentes de transporte terrestre principalmente em virtude da pouca experiência no trânsito ${ }^{23,24,25}$, fato esse que foi reafirmado nesse estudo, pois foi observado que $65,0 \%$ dos indivíduos avaliados conduziam moto há menos de quatro anos. Esse resultado é condizente com estudo de Debieux et al (26) que também revelou um maior número de acidentados em pessoas cuja experiência em conduzir motos era inferior a 5 anos $(67,0 \%)$.

Porém, outros fatores também podem justificar essa questão, como a necessidade de autoafirmação perante os grupos, o excesso de velocidade e o desrespeito às normas de segurança ${ }^{24,25}$. Nos jovens, a tomada de decisão é marcada pela impulsividade, ousadia e confiança excessiva em sua própria destreza22,27.

Apesar disso, foram as mulheres que apresentaram piores escores na LEFS, ou seja, do ponto de 
Tabela 3- Relação entre as variáveis independentes e o escore LEFS, Natal, RN, Brasil, 2011.

\begin{tabular}{|c|c|c|c|c|}
\hline & & $\begin{array}{l}\text { ESCORE } \\
\text { Média }\end{array}$ & $\begin{array}{l}\text { FS } \\
\pm \mathrm{dp}\end{array}$ & $\mathbf{p}$ \\
\hline \multirow[t]{2}{*}{ Sexo } & Masculino & 34,72 & 18,48 & 0,004 \\
\hline & Feminino & 21,31 & 11,18 & \\
\hline \multirow[t]{2}{*}{ Estado Civil } & $\begin{array}{l}\text { Casado/união } \\
\text { estável }\end{array}$ & 32,80 & 21,40 & 0,65 \\
\hline & $\begin{array}{l}\text { Viúvo/ } \\
\text { solteiro,divorciado }\end{array}$ & 30,42 & 15,77 & \\
\hline \multirow[t]{2}{*}{ Habilitação } & Sim & 33,1 & 18,8 & \multirow{2}{*}{0,45} \\
\hline & Não & 29,3 & 16,7 & \\
\hline \multirow[t]{2}{*}{ Revisões } & Sim & 34,43 & 18,80 & 0,001 \\
\hline & Não & 16,00 & 3,37 & \\
\hline \multirow[t]{2}{*}{ Fez cirurgia } & Sim & 31,49 & 17,26 & 0,68 \\
\hline & Não & 29,08 & 19,85 & \\
\hline \multirow[t]{2}{*}{ Fez fisioterapia } & Sim & 32,67 & 15,38 & 0,50 \\
\hline & Não & 29,30 & 17,72 & \\
\hline \multirow[t]{2}{*}{ Tem sequela } & $\operatorname{Sim}$ & 27,77 & 13,70 & 0,007 \\
\hline & Não & 57,57 & 20,19 & \\
\hline \multirow[t]{2}{*}{ Acidente anterior } & $\operatorname{Sim}$ & 35,45 & 18,62 & 0,20 \\
\hline & Não & 28,81 & 17,14 & \\
\hline \multirow[t]{2}{*}{ Uso capacete } & Sim & 31,89 & 18,47 & 0,62 \\
\hline & Não & 28,50 & 14,57 & \\
\hline \multirow[t]{2}{*}{ Passageiro } & Sim & 32,55 & 21,34 & 0,70 \\
\hline & Não & 30,63 & 15,59 & \\
\hline \multirow[t]{2}{*}{ Álcool } & Sim & 26,75 & 11,44 & 0,59 \\
\hline & Não & 31,75 & 18,30 & \\
\hline \multirow[t]{2}{*}{ Ano moto } & Mais de 4 anos & 30,57 & 20,96 & 0,78 \\
\hline & Menos de 4 anos & 32,27 & 16,74 & \\
\hline \multirow[t]{2}{*}{ Potência moto } & Menos de 125 cc & 32,93 & 18,37 & 0,48 \\
\hline & Mais de 125 cc & 28,54 & 18,97 & \\
\hline \multirow[t]{2}{*}{ Outros veículos } & Não & 28,36 & 15,25 & 0,30 \\
\hline & Sim & 33,57 & 19,48 & \\
\hline \multirow[t]{2}{*}{ Hora acidente } & Manhã/tarde & 31,56 & 17,82 & 0,91 \\
\hline & Noite & 31,00 & 18,39 & \\
\hline
\end{tabular}

Tabela 4- Resultado da análise de regressão linear múltipla com as variáveis preditivas do escore da LEFS, Natal, RN, Brasil, 2011.

\begin{tabular}{llll}
\hline \hline VARIÁVEIS & $\beta$ & ERRO-PADRÃO & p \\
\hline Sexo & $-11,1$ & 5,1 & 0,02 \\
Sequela física & 22,9 & 6,1 & 0,001 \\
Tempo do acidente & 2,4 & 1,22 & 0,04 \\
\hline \hline
\end{tabular}


vista funcional, o acidente repercutiu de forma mais grave nos seus membros inferiores. Segundo o levantamento realizado pelo Instituto de Pesquisa Econômica Aplicada ${ }^{28}$, apesar dos homens estarem envolvidos em maior número nos acidentes, as muIheres estão mais presentes nos acidentes considerados graves.

Considerando-se as variáveis quantitativas como tempo de habilitação, tempo para cirurgia, tempo de internação, tempo para inicio da fisioterapia após a cirurgia e o tempo entre o acidente e a avaliação, apenas esta última apresentou significância com os escores de funcionalidade da escala LEFS. Entretanto, o baixo valor de correlação encontrado $(r=0,39)$ demonstra que apesar de haver uma correlação positiva, o mesmo foi apenas regular.

Pode-se observar também que aqueles que apresentaram sequelas físicas provenientes do acidente tiveram uma pior funcionalidade de forma estatisticamente significante. Isto pode estar relacionado à falta de informação e/ou orientação a respeito de um programa fisioterapêutico adequado e precoce principalmente no período pós-hospitalar, pois como foi visto nos resultados desse estudo, $64,7 \%$ dos pacientes não haviam realizado fisioterapia após o trauma, e aqueles que a fizeram, demoraram em média 60 dias para iniciá-la. Além disso, pode favorecer este quadro a presença de instabilidade, fraqueza e restrição de mobilidade, bem como os distúrbios de sensibilidade e/ou o desconforto com as cicatrizes.

Em estudo realizado em Santa Catarina, que objetivava verificar a incidência de atendimentos fisioterapêuticos prestados aos pacientes que sofreram fraturas de diversas etiologias, entre elas acidentes motociclísticos, no período hospitalar e pós-hospitalar, mostrou que apenas $13,7 \%$ dos pacientes realizaram fisioterapia hospitalar e quanto ao encaminhamento destes para a fisioterapia pós-alta hospitalar, o referido estudo não encontrou registros ${ }^{29}$.

Essa situação pode gerar um considerável problema de saúde em relação à recuperação dos pacientes, pois se tem conhecimento do importante papel da fisioterapia no tratamento de pacientes fraturados, por agir como um complemento e não como um fator único. Por isso, os bons resultados no tratamento de fratura dependem muito da reabilitação. Em- bora todo paciente adulto com fratura importante deva fazer exercício supervisionado com a maior frequência possível, ele deve ser alertado de que esse tratamento é apenas uma parte da reabilitação e que o resultado final depende muito da continuidade das atividades fora das sessões de fisioterapia. Foi também comprovado que a fisioterapia quando executada de forma precoce no atendimento do paciente crítico politraumatizado, é fundamental, uma vez que a taxa de mortalidade pode ser reduzida com a intervenção dos profissionais dessa área ${ }^{30}$.

Outro fator determinante estatisticamente para a funcionalidade de membros inferiores foi o tempo do acidente. Pacientes que haviam sofrido fratura há mais tempo apresentaram melhor funcionalidade que aqueles que haviam sofrido há menos tempo. Isto pode ser evidenciado a partir da premissa de que pacientes com lesões mais tardias e que realizaram um tratamento adequado no período pós-trauma já haviam adquirido certa estabilidade osteo-músculo-tendínea. Em estudo realizado por Itami et al. ${ }^{22}$, foi verificado que ao avaliar independência funcional através da Medida de Independência Funcional (MIF), em pacientes com fraturas, quando analisou-se funcionalidade em relação ao domínio motor da escala, houve um aumento considerável na média dos valores da MIF entre o momento da alta hospitalar e um mês após o regresso ao domicílio do paciente, sugerindo ganho de independência funcional. No caso do presente estudo, a avaliação foi realizada de um mês até seis meses após o evento, o que ratifica ainda mais essa questão.

Em relação aos pacientes com menor tempo de lesão que estavam, portanto, na fase aguda, estes apresentaram uma pior funcionalidade. Este dado corrobora com esse mesmo estudo ${ }^{22}$, o qual também verificou que em relação ao MIF motor, citado anteriormente, as atividades referentes a esse domínio estariam bastante afetadas no período de trauma agudo; tendo em vista que das 13 atividades avaliadas, oito dependem direta ou indiretamente de uma boa funcionalidade de membros inferiores para que possam ser executadas sem o auxílio de terceiros e/ ou maior demanda de tempo.

As limitações desse estudo consistiram no pequeno número amostral, o qual foi prejudicado pela não 
existência, muitas vezes, de um número telefônico dos pacientes nos boletins de urgência/emergência verificados, pela não especificação dos médicos plantonistas do quadro clínico dos pacientes e pela dificuldade em localizar os sujeitos por telefone.

\section{CONCLUSÃO}

Foi evidenciado nesse estudo que vítimas de acidentes motociclísticos com fratura em membros inferiores obtiveram piores escores de funcionalidade quando apresentaram sequelas físicas pós-trauma, eram do sexo feminino e com pouco tempo de acidente.

Nesse sentido, se faz necessário uma maior atenção aos pacientes em relação a um tratamento tanto clínico quanto fisioterapêutico adequado, principalmente na fase pós-hospitalar. Tendo em vista que muitos pacientes por não receberem as informações necessárias, muitas vezes não tem um período de reabilitação eficaz, podendo assim tornarem-se indivíduos com comprometimento de suas capacidades funcionais. Não obstante, é importante enfatizar o desenvolvimento de mais programas de conscientização no trânsito, destinados aos motociclistas, maior fiscalização nas vias públicas e punição adequada aos infratores.

Faz-se necessário também a realização de mais estudos relacionados ao tema da funcionalidade em acidentados com motocicletas, pois este ainda encontra-se bastante escasso na literatura, e também com a participação de amostras maiores da população.

\section{REFERÊNCIAS}

1. Oliveira NLB, Souza RMC. Diagnóstico de lesões e qualidade de vida de motociclistas, vítimas de acidentes de trânsito. Rev Latino-am Enferm. 2003; 11: 749-56.

2. Bastos YGL, Andrade SM, Soares DA. Características dos acidentes de trânsito e das vítimas atendidas em serviço pré-hospitalar em cidade do Sul do Brasil, 1997/2000. Cad Saúde Pública. 2005; 21: 815-22.

3. Bachierri G, Barros AJD. Acidentes de trânsito no Brasil de 1998 a 2010: Muitas mudanças e poucos resultados. Rev. Saúde Pública. 2011; 45: 949-63.

4. Andrade SM, Mello-Jorge, MHP. Acidentes de transporte terrestre em município da Região Sul do Brasil. Rev. Saúde Pública. 2001; 3: 318-20.
5. Pligher FA. Arte, Mito e Tecnologia: A motocicleta como fenômeno cultural do século XX (Dissertação). Campinas: Universidade Estadual de Campinas, UNICAMP, 2006.

6. Oliveira NLB, Souza RMC. Retorno a atividade produtiva de motociclistas vítimas de acidentes de trânsito. Acta Paul Enferm. 2006; 19: 284-289.

7. Brasileiro BF, Vieira JM, Silveira CES. Avaliação de traumatismos faciais por acidentes motociclisticos em Aracaju/SE. Rev circ traumatol Buco-maxilo-facial. 2010; 2: 97-104.

8. Rodrigues NB, Gimenes GM, Lopes CA, Rodrigues JMS. Mortes, lesões e padrões das vítimas em acidentes de trânsito com ciclomotores no município de Sorocaba, São Paulo, Brasil. Rev Fac Ciênc Méd. 2010;12: 21-25.

9. Montenegro MMS, Duarte EC, Prado RR, Nascimento AF. Mortalidade de motociclistas em acidentes de transporte no Distrito Federal, 1996 a 2007. Rev Saúde Pública. 2011; 45: 529-538.

10. Koizumi MA. Padrão das lesões nas vítimas de acidentes de motocicletas. Rev. Saúde Púb. 1992; 26 (5): 306 315.

11. Instituto de Pesquisa Econômica Aplicada. Pesquisa sobre custos de acidentes de trânsito em rodovias brasileiras [Internet] 2009. Disponível em: http:// www. ipea.gov.br.

12. Santos AMR, Moura MEB, Nunes BMVT, Leal CFS, Teles JBM. Perfil das vítimas de trauma por acidente de moto atendidas em um serviço público de emergência. Cad. Saúde Pública, Rio de Janeiro. 2008; 24: $1927-$ 1938.

13. Sado MJ, Morais FD, Viana FP. Caracterização das vítimas por acidentes motociclísticos internadas no hospital de urgência de Goiânia. Rev. Movimenta. 2009; 2 (2): 49-53.

14. Ganne N. Estudo sobre acidentes de trânsito envolvendo motocicletas na Cidade de Corumbá e região, Estado do Mato Grosso do Sul, Brasil, no ano de 2007. Rev Pan-Amaz Saúde. 2010; 1(3): 19-24.

15. Gawryszewski VP, Coelho HMM, Scarpelini S, Zan R, Mello Jorge MHP, Rodrigues EMS. Perfil dos atendimentos a acidentes de transporte terrestre por serviços de emergência em São Paulo, 2005. Rev. Saúde Pública. 2009; 43: 275-82.

16. Calil AM, Sallun EA, Domingues CA, Nogueira LS. Mapeamento das lesões em vítimas de acidentes de trânsito: Revisão sistemática da literatura. Rev latino-am Enfermagem. 2009; 17 (1): 120-125.

17. Milcheski DA, Ferreira MC, Nakamoto HA, Tuma P Jr Gemperli R. Degloving injuries of lower extremity-proposal of a treatment protocol. Rev Col Bras Cir. 2010; 37:199-203.

18. Binkley JM, Stratford PW, Lott AS, Ridlle DL. The Lower Extremity Functional Scale (LEFS). Scale Development, Measurement, Properties and Clinical Aplications. Phys Ther. 1999; 79(4): 371-383.

19. Talving P, Teixeira PG, Barmparas G, Dubose J, Preston C, Inaba K, Demetriades D. Motorcycle-related inju- 
ries: effect of age on type and severity of injuries and mortality. J Trauma. 2010; 68: 441-446.

20. Parreira JG, Gregorut F, Perlingeiro JA, Solda SC, Assef JC. Comparative analysis of injuries observed in motorcycleriders involved in traffic accidents and victims of other blunt trauma mechanisms. Rev Assoc Med Bras. 2012; 58:76-81.

21. Hatamabadi $H$, Vafaee R, Hadadi M, Abdalvand A, Esnaashari $\mathrm{H}$, Soori $\mathrm{H}$. Epidemiologic study of road traffic injuries by road user type characteristics and road environment in Iran: a community-based approach.Traffic Inj Prev. 2012; 13: 61-64.

22. Itami LT, Faro ACM, Meneguin P, Leite RCBO, Silveira CT. Adultos com fraturas: das implicações funcionais e cirúrgicas a educação em saúde. Rev Esc Enferm USP. 2009; 43: 1238-1243.

23. Coben JH, Steiner CA, Owens P. Motorcycle-related hospitalizations in the United States, 2001. Am J Prev Med. 2004; 27: 355-362.

24. Moreira TS, Sabino GS, Resende MA. Instrumentos clínicos de avaliação funcional do tornozelo: revisão sistemática. Fisioterapia e Pesquisa. 2010; 17(1): 8893.
25. Andrade SM, Soares DA, Braga GP, Moreira JH, BoteIho FMN. Comportamento de risco para acidentes de trânsito: um inquérito entre estudantes de medicina na região sul do Brasil. Rev Assoc Med Bras. 2003; 49: 439-444.

26. Debieux P, Chertman C, Mansur NSB, Dobashi E, Fernandes HJA. Lesões do aparelho locomotor nos acidentes com motocicleta. Acta Ortop. Bras. 2010; 18:353-356.

27. Marín-Leon L, Vizzoto MM. Comportamentos no trânsito: um estudo epidemiológico com estudantes universitários. Cad. Saúde Pública. 2003; 19: 515-523.

28. Instituto de Pesquisa Econômica Aplicada. Metodologia para estimativa de custos de cuidados com saúde: Análise dos fatores de gravidade e recomendações para redução dos custos. Brasília, Julho de 2008.

29. Zago APV, Grasel CE, Padilha JA. Incidência de atendimentos fisioterapêuticos em vítimas de fratura em um hospital universitário. Fisioter Mov. 2009; 22: 565-573.

30. Adams JC, Hamblen DL. Manual de fraturas. São Paulo: Artes Médicas; 1994 